\title{
Lipoic Acid Metabolism as a Potential Chemotherapeutic Target Against Plasmodium falciparum and Staphylococcus aureus
}

\author{
Sun Liu Rei Yan ${ }^{1}$, Felipe Wakasuqui ${ }^{1}$, Xiaochen $D u^{2}$, Matthew R. Groves ${ }^{2 *}$ and \\ Carsten Wrenger ${ }^{1 *}$
}

${ }^{1}$ Unit for Drug Discovery, Department of Parasitology, Institute of Biomedical Sciences-ICB, University of São Paulo, São Paulo, Brazil, ${ }^{2}$ Structural Biology in Drug Design, Department of Drug Design, Groningen Research Institute of Pharmacy, University of Groningen, Groningen, Netherlands

\section{OPEN ACCESS}

Edited by:

Siva S. Panda,

Augusta University, United States

Reviewed by:

Tung Truong,

University of Pittsburgh, United States

Assem Barakat,

King Saud University, Saudi Arabia

Alexander Kastaniotis,

University of Oulu, Finland

*Correspondence:

Matthew R. Groves

m.r.groves@rug.nl

Carsten Wrenger

cwrenger@icb.usp.br

Specialty section: This article was submitted to Medicinal and Pharmaceutical

Chemistry,

a section of the journa

Frontiers in Chemistry

Received: 15 July 2021 Accepted: 21 October 2021 Published: 04 November 2021

Citation:

Rei Yan SL, Wakasuqui F, Du X, Groves MR and Wrenger C (2021)

Lipoic Acid Metabolism as a Potential

Chemotherapeutic Target Against

Plasmodium falciparum and

Staphylococcus aureus.

Front. Chem. 9:742175.

doi: 10.3389/fchem.2021.742175
Lipoic acid (LA) is an organic compound that plays a key role in cellular metabolism. It participates in a posttranslational modification (PTM) named lipoylation, an event that is highly conserved and that occurs in multimeric metabolic enzymes of very distinct microorganisms such as Plasmodium sp. and Staphylococcus aureus, including pyruvate dehydrogenase $(\mathrm{PDH})$ and $\alpha$-ketoglutarate dehydrogenase $(\mathrm{KDH})$. In this mini review, we revisit the recent literature regarding LA metabolism in Plasmodium $s p$. and Staphylococcus aureus, by covering the lipoate ligase proteins in both microorganisms, the role of lipoate ligase proteins and insights for possible inhibitors of lipoate ligases.

Keywords: Lipoic acid, malaria, protein lipoylation, Plasmodium, S. aureus, Lipoic acid (LA), lipoylation

\section{INTRODUCTION}

LA \{6,8-dithiooctanoic acid or 5-[(3R)-dithiolan-3-yl]pentanoic acid $\}$ is an organosulfur compound (Figure 1B and Figure 1C) that has long been reported for antioxidant effects and potential therapeutic benefits in treating a variety of diseases, such as neurodegenerative diseases, diabetes, and cardiovascular conditions (Marangon et al., 1999; Amom et al., 2008; McNeilly et al., 2011; Tromba et al., 2019; Li et al., 2020; Molz et al., 2021). In addition to its potential therapeutic effects and current use as a potential antioxidant in dietary supplementation, LA is an essential cofactor for many enzymatic reactions in key biochemical pathways. To date, LA is known to act as a cofactor in five different enzyme complexes: the glycine cleavage system (GCS), pyruvate dehydrogenase (PDH), $\alpha$-ketoglutarate dehydrogenase (KGDH), branched-chain $\alpha$-keto acid dehydrogenase (BCDH), and acetoin dehydrogenase (AoDH) (Oppenheim et al., 2014). The $\alpha$-ketodehydrogenase-complexes contain three protein subunits, named E1, E2, and E3. LA in the free form of lipoate is attached to the E2 lysine residues or to the H protein of the GCS. Lipoate metabolism is present across different human pathogens, including Plasmodium sp., the causative agent of malaria, a tropical disease that was responsible for about 229 million cases worldwide in 2019 only, with an estimated 409,000 deaths in the same year (World Health Organization, 2020). Although more knowledge on lipoylation has been gained (Cao et al., 2018a; Laczkovich et al., 2018; Zhang et al., 2020; Tang et al., 2021), it remains an attractive topic to better understand the metabolic consequences of dysregulated lipoylation and how LA metabolism enzymes could be explored as a potential drug target in different diseases. From a chemical perspective, the disulfide bond in oxidized/reduced form provides a strong redox couple that is important for reactive oxygen species (ROS) scavenging and 


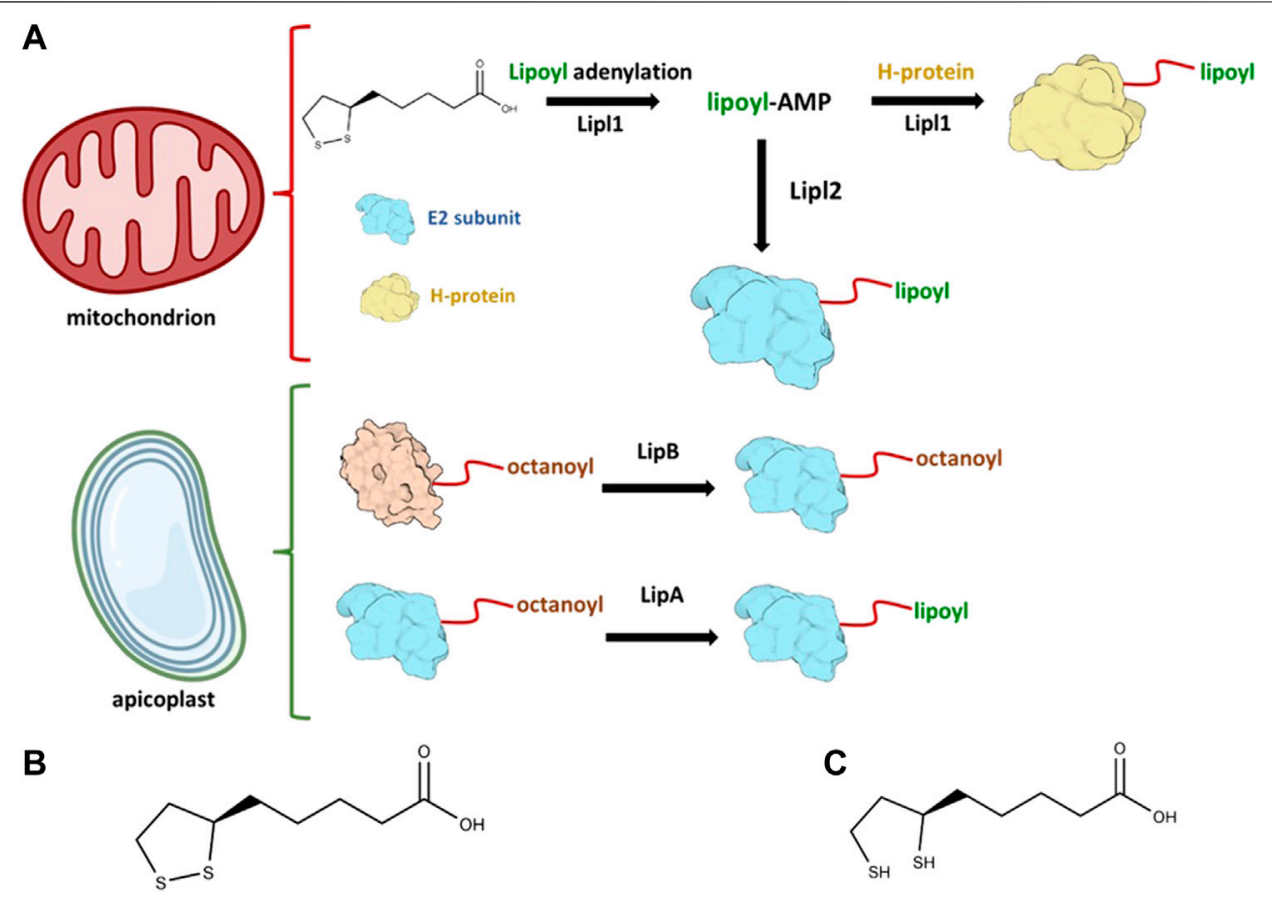

FIGURE 1 | Lipoylation in Plasmodium falciparum occurs in both mitochondrion and apicoplast. In (A) the enzymatic reactions that takes place in each of these compartments are illustrated. The generation of lipoyl-AMP is needed to activate Lipl2 and lipoylate the E2 subunit of both BCDH and KDH. The oxidized lipoate is also attached to the $\mathrm{H}$-protein of the parasite. Oxidized lipoic acid is shown in (B) and reduced lipoic acid is shown in (C), Illustrations were created with BioRender.com (License \#2364-1,511, Toronto, ON, Canada).

for the redox-dependent reactions that regulate multienzyme complexes. Examples of scavenged ROS include peroxynitrite (ONOO-), hypochlorous acid ( $\mathrm{HClO})$, peroxyl radical (ROO.), and hydroxyl radicals $(\cdot \mathrm{OH})$. However, evidence so far indicates that hydrogen peroxide $\left(\mathrm{H}_{2} \mathrm{O}_{2}\right)$ is not directly scavenged by LA (Xiao et al., 2012). LA can also act as a chelator of $\mathrm{Cu}^{2+}, \mathrm{Pb}^{2+}$ and $\mathrm{Zn}^{2+}$ in vitro. Therefore, LA could be potentially a treatment for diseases where these metals may play an important role in their progression (Xiao et al., 2012; Hane and Leonenko, 2014; Smirnova et al., 2018).

\section{Lipoylation in Plasmodium falciparum}

Antimalarial drug resistance still is a major health concern worldwide and poses a real threat for the control of malaria (WHO, 2020). Two main species are responsible for the majority of malaria cases worldwide: $P$. vivax represents $75 \%$ of malaria cases in the Americas, while $99.7 \%$ of estimated malaria cases in Africa were caused by P. falciparum (World Health Organization, 2021). Lipoylation of Plasmodium proteins is an event that occurs in two different compartments of the parasite: mitochondrion and the apicoplast, a unique Apicomplexan plastid organelle that evolved from endosymbiotic events. The parasite relies both on lipoate biosynthesis and lipoate-scavenged pathways: lipoate biosynthesis takes place in the apicoplast, while scavenged lipoate from host is metabolized in the mitochondrion.

Two important P. falciparum lipoate ligases, Lipl1 and Lipl2, are known to play key roles in protein lipoylation. Lipl1 is responsible for the lipoylation of $\mathrm{GcvH}$ protein by employing the oxidized form of lipoate and Lipl2 is responsible for the lipoylation of the E2 subunit from $\mathrm{BCDH}$ and $\mathrm{KDH}$. However, Lipl2 depends on the formation of dihydrolipoyl-AMP in reducing conditions to transfer the lipoyl moiety to the $\mathrm{N}$-lysine residue, thereby impacting the activity of Lipl1 in a reducing environment. In oxidative decarboxylation reactions of $\alpha$-ketoacid complexes, lipoate derived from LA plays a key role as a cofactor. The $\alpha$-ketoacids complexes are formed by three different subunits, named E1, E2, and E3. In the case of the glycine cleavage complex (GCV), the H-protein $(\mathrm{GcvH})$ serves as the lipoyl domain for lipoate. All three proteins-GcvH, E2 subunit of both $\mathrm{BCDH}$ and $\mathrm{KDH}(\mathrm{E} 2-\mathrm{BCDH}$ and $\mathrm{E} 2-\mathrm{KDH}$, respectively)-are lipoylated through lipoate covalent ligation to the lysine residue at the $\mathrm{N}$-terminus of lipoate domain and they are known to be localized in the parasite mitochondrion (Afanador et al., 2014). In vitro lipoylation assays show that Lipl1 is required for the lipoylation of $\mathrm{GcvH}, \mathrm{E} 2-\mathrm{BCDH}$ and E2$\mathrm{KDH}$ (Afanador et al., 2014). Evidence so far indicates that the role of Lipl2 in P. falciparum is to act as a lipoyl-AMP: $N^{\varepsilon}$-lysine lipoyltransferase. The intermediate lipoyl-AMP generated by Lipl1 is employed by Lipl2 to lipoylate both E2-BCDH and $\mathrm{E} 2-\mathrm{KDH}$. Therefore, the catalytic activity of Lipl2 also depends on the activity of Lipl1, since only Lipl1 can generate lipoyl-AMP conjugate (Afanador et al., 2014). Recently, Leung and colleagues et al (Leung et al., 2021) discussed the role of GcvH beyond GCV system, such as its possible role in the lipoylation of $\alpha$-ketoacids dehydrogenase proteins. Although recent work has been performed to better elucidate the LA metabolism in eukaryotes 
A

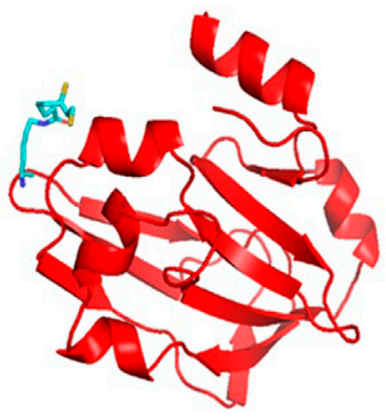

C

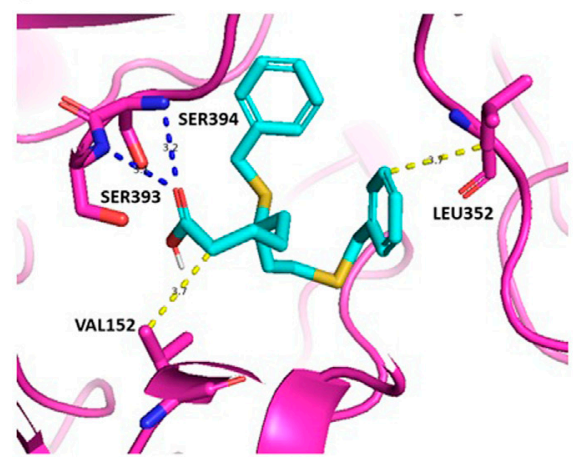

B

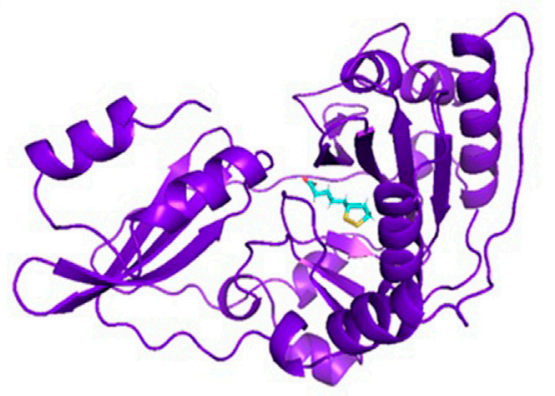

D

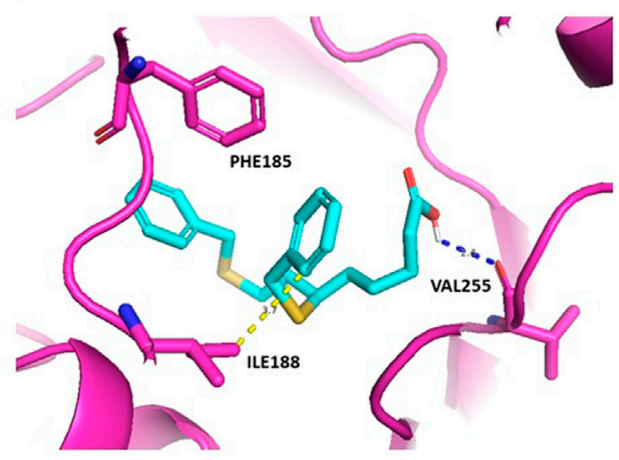

FIGURE 2 | Homology models for S. aureus GcvH protein (A) and Lipl1 protein (B). Lipoate moiety shown in stick representation with PyMOL. PDB access numbers: $3 A B 9$ and 5T8U. Predicted three-dimensional structure for LipA of $P$ falciparum with devimistat. The three dimensional structure of LipA was obtained from AlphaFold (PDB access number: Q8IDQ0), the search for a predicted active site was performed using the package FtMap (Kozakov et al., 2015). Based upon the two predicted binding sites docking was performed using Autodock/sMina. The lowest energy poses are shown in (C) and (D) for each predicted binding site. Potential hydrophobic interactions are shown by dotted yellow lines with the indicated residues, blue dotted lines represent hydrogen bonds; pi stacking: PHE185. In (C), the calculated binding energy is of $-7.312 \mathrm{kcal} / \mathrm{mol}$ and, in (D), the calculated binding energy is of $-7.075 \mathrm{kcal} / \mathrm{mol}$.

(Cao et al., 2018a; Vacchina et al., 2018; Biddau et al., 2021; Pietikäinen et al., 2021), there are still unanswered questions, such as why there are two protein ligases instead of only one protein ligase in Plasmodium and the role of lipoylated proteins in the parasite. Lipoate scavenging for use in the mitochondrion remains an open research topic to be elucidated. Scientific evidence so far indicates that lipoate scavenged from the host is important for $P$. falciparum erythrocytic stage parasites (Günther et al., 2009a). Murine malaria models and human malaria model show that lipoylation may play an essential role in Plasmodium (Wang et al., 2017). In particular, an experiment in which LA analogues were utilized (Deschermeier et al., 2012) showed decreased mitochondrial lipoylation and inhibition of parasite growth. A general scheme of how proteins are lipoylated in P. falciparum is briefly described in Figure 1A. Lipl1 is known to be a mitochondrial protein while Lipl2 is both mitochondrial and found in the apicoplast, a unique plastid organelle found in Apicomplexan parasites. This organelle is important in Plasmodium sp. due to the presence of important parasite metabolic pathways, such as the synthesis of isopentenyl diphosphate (IPP) (Wiley et al., 2015), a precursor of isoprenoids, the type II fatty acid synthesis (FAS-II) (van Schaijk et al., 2014), and the lipoate biosynthesis that is mediated by two different enzymes: octanoyl-ACP:protein
$N$-octanoyltransferase (LipB) and lipoyl synthase (LipA) (Wrenger and Müller, 2004). LipB and LipA orchestrate the biosynthesis of LA in the apicoplast: LipB is responsible for the transfer of the octanoyl-moiety to the E2 subunit whereas LipA acts as a catalyst for the insertion of two sulfurs at positions C6 and $\mathrm{C} 8$ of the octanoyl-moiety that is bound to the E2 subunit of the PDH in the apicoplast.

Recently, Biddau and collaborators (Biddau et al., 2021) provided more evidence on the putative role of LA in redox regulation. Using a $N$-octanoyltransferase (LipB) P. falciparum 3D7 knockout strain, the authors identified upregulation of antioxidant-related cytosolic proteins that could be related to plastid-cytosol signaling. Additionally, experiments in Anopheles mosquitoes that are the vector for malaria transmission indicated that LipB knockout parasites could not produce salivary gland sporozoites, possibly indicating the need of LA synthesis in the apicoplast for the full development of $P$. falciparum in Anopheles.

In terms of drug discovery, the lack of the structural information available for LipA in Plasmodium sp. may also require in silico predictions, as the one available from Alphafold (Jumper et al., 2021). We used the FTMap web servers (Kozakov et al., 2015) for predicting protein binding hot spots through computational approaches, which shows 
that there are two potential hot spots, cluster 1 and cluster 2 [Figure 2C and 2(d)]. Devimistat, an LA analogue drug candidate, was chosen for investigation as a potential inhibitor compound. Molecular docking using AutoDock/Smina (Koes et al., 2013) was used to predict the binding-conformation of devimistat to LipA. The result shows devimistat bound to lipoic acid protein at the catalytic site of the homologous $\alpha$-ketoglutarate dioxygenase of $E$. coli (RCSB: 1GY9, Figure 2C and 2(d). For cluster 1 (Figure 2C), there are two hydrogen bonds between serine 394, serine 393 and a compound carboxy groups hydrophobic interaction between leucine 352 and the compound 8-phenyl group, valine 152 and the compound 2carbon atom. For cluster 2 (Figure 2D), there is a hydrogen bond between valine 255 and a compound carboxy functional group; hydrophobic interaction between isoleucine 188 and the compound 6-phenyl group; pi stacking between phenylalanine 185 and the compound 6,8-diphenyl groups.

\section{Fatty Acid Synthesis (FAS-II) and Iron-Sulfur (Fe-S) Clusters in Plasmodium sp.}

Lipids are required for Plasmodium growth and replication. The FAS-II pathway is present in the malaria parasite apicoplast, specifically in sporozoite and liver stages. Vaughan and colleagues et al. Vaughan et al. (2009) demonstrated the importance of FASII for the parasite when migrating from liver to the blood asexual stage, both in P. yoelli and P. falciparum. Later it was also shown (van Schaijk et al., 2014) that FAS-II is required for midgut oocyst sporozoite production during the Anopheles mosquito stage of $P$. falciparum life cycle, but dispensable in rodent malaria models of $P$. yoelli and $P$. berghei. Thus, much research has been conducted to explore inhibitory approaches in the late-liver parasite development. While initial FAS-II inhibitors eliminated blood-stage malaria parasites, their mode of action were shown to be off-target (McFadden and Yeh, 2017). Most of these drugs act in the blood stage of the parasite cycle, yet FAS-II is not essential in that stage. Therefore, tackling the parasite at liver stage is likely to be a more promising avenue for drug development. In terms of lipoylation of $P$. falciparum proteins, the FAS-II pathway in the apicoplast has the fundamental role of providing the octanoyl-acyl carrier protein (octanoyl-ACP) as a precursor for the generation of de novo LA (Wrenger and Müller, 2004; Shears et al., 2015).

In Plasmodium, the importance of $\mathrm{Fe}-\mathrm{S}$ clusters for intraerythrocytic stage growth is well established. Fe-S clusters are found in different forms, such as $4 \mathrm{Fe}-4 \mathrm{~S}$. These clusters can act as cofactors and bind proteins via cysteine residues. Two proteins of the $P$. falciparum sulfur mobilization pathway (SUF) were characterized (Charan et al., 2014): PfSufS and PfSufE. The first has cysteine desulfurase activity while the latter enhances the activity of PfSufS. Both proteins mediate sulfur mobilization, which is the first step in the apicoplast SUF pathway, and both are localized in the apicoplast. Since SUF is not present in humans, the enzymes of this pathway are attractive targets for parasite inhibition and eventually the validation of this pathway for druggability purposes. More recently, functional experiments performed with $P$. vivax in clinical isolates show that the SUF pathway is conserved as in laboratory strains (Pala et al., 2019). As briefly described in this mini review, the lipoate synthase (LipA) is an example of an enzyme that depends on $4 \mathrm{Fe}-4 \mathrm{~S}$ clusters. It is localized in the apicoplast, where it uses the iron sulfur clusters to add sulfur atoms onto carbons $\mathrm{C} 6$ and $\mathrm{C} 8$ of the octanoic acid, thereby completing the lipoate synthesis on the PDH E2 subunit (Thomsen-Zieger et al., 2003; Wrenger and Müller, 2004; Günther et al., 2009b; Storm and Müller, 2012; Shears et al., 2015).

\section{LA Metabolism in Staphylococcus aureus}

Staphylococcus aureus is a gram-positive bacterium able to colonize human skin and mucous membranes, living as a commensal in healthy individuals. However, it is also capable of invasion, causing several clinical manifestations. It is a leading cause of endocarditis, bacteremia, osteomyelitis and skin and soft tissue infections (Turner et al., 2019). The greatest concern with $S$. aureus is its developed multi drug resistance and persistent high mortality (van Hal et al., 2012).

Like in $P$. falciparum, $S$. aureus has both de novo biosynthetic pathway and salvage pathway for the generation of LA. With the exception of $B$. anthracis, no other pathogenic Firmicutes has such a diversity of enzymes involved in the acquisition of this cofactor (Spalding and Prigge, 2010). S. aureus has two lipoateprotein ligases, named LplA1 and LplA2, for salvaging LA and octanoic acid from the environment (Zorzoli et al., 2016; Cao et al., 2018b). The attachment of free LA in S. aureus occurs via LplAs in a two-step reaction: first there is the formation of the intermediate lipoyl-AMP in the presence of $\mathrm{Mg}^{2+}$ and ATP, followed by the binding of the lipoyl group to the apoprotein (Fujiwara et al., 2005).

LplA1 binds LA mainly to GcvH, while LplA2 binds LA to the E2 subunits of $\alpha$-ketoacid dehydrogenases, as well as to the operon-linked GcvH-like protein, GcvH-L (Laczkovich et al., 2018). GcvH and GcvH-L thereby provide storage of lipoylated proteins. In vivo studies using mouse infection models of LplA1 or LplA2 knockouts demonstrated that the presence of either enzyme is enough to promote kidney infection (Zorzoli et al., 2016). LplA2 is encoded in an operon together with an ADP-ribosyltransferase, macrodomain protein, luciferase-like monooxygenase and the protein GcvH-L, which suggests that LplA2 may participate in LA-dependent maintenance of redox homeostasis (Rack et al., 2015). Illustration of homology models for $S$. aureus $\mathrm{GcvH}$ and LplA1 proteins with lipoate moiety is shown in Figures 2A, B

Biosynthesis of LA begins when the fatty acid intermediate octanoyl is transferred from an acyl carrier protein to the $\mathcal{E}$-amino group of a lysine in the lipoyl domain of the GcvH by the enzyme LipM. (Douglas et al., 2006; Zorzoli et al., 2016). The octanoyl moiety is sulfurized into lipoyl by the enzyme LipA. LipA is a member of the radical $S$-adenosyl-L-methionine (SAM) enzymes (Christensen and Cronan, 2010), which use a [4Fe-4S] cluster as an electron donor to reductively cleave SAM, generating a deoxyadenosyl radical and methionine. Two 5-deoxyadenosyl break $\mathrm{C}-\mathrm{H}$ bond in position 6 and 8 of the octanoyl moiety, creating carbon radicals that allow sulphur insertions, with the 
auxiliary iron-sulphur cluster of LipA acting as a sulphur donor (Douglas et al., 2006). Finally, S. aureus possess the enzyme LipL, an amido transferase responsible for the transfer of octanoyl or lipoyl groups between $\mathrm{GcvH}, \mathrm{GcvH}$-like protein ( $\mathrm{GcvH}-\mathrm{L})$ and $\alpha$-keto dehydrogenases, as well as inter E2-subunits. This flexibility gives $S$. aureus robust resources to supplement its requirements for LA (Teoh et al., 2019).

LA biosynthesis and its salvage pathway plays a major role in facilitating the pathogenesis of microorganisms, promoting pathogen survival within an infected host (Spalding and Prigge, 2010). S. aureus has specific requirements during infection, where de novo biosynthesis of LA is necessary to infect the heart and salvage is required for infection of the kidney (Zorzoli et al., 2016). LipL was shown to be necessary within the host, but not necessary at skin sites, where $S$. aureus can overcome its need for branched-chain fatty acids by scavenging unsaturated fatty acids from host skin. (Teoh et al., 2021). In addition, lipoyl-E2-PDH secreted in the extracellular environment blunts activation of macrophage toll-like 1/2 receptors (Grayczyk et al., 2017) and reduces the generation of ROS and RNS by macrophage NADPH oxidase and iNOS (Grayczyk and Alonzo, 2019), which reinforces the importance of lipoylation in the context of infection.

\section{LA Metabolism in Humans and Other Organisms}

As mentioned above, lipoylation is a PTM event that occurs in different organisms. Good drug targets must have minimal effects on the host or at least mitigation of off-target effects. The catalysis of LA assembly in human was recently elucidated by Cao and colleagueset al. (Cao et al., 2018a), reporting the relevant LIPT1 and LIPT2 enzyme activities: LIPT1 catalyzes the attachment of the lipoyl moiety to the lipoyl domain of the protein, acting as a lipoyl amidotransferase, while LIPT2 acts as an octanoyltransferase. Both

\section{REFERENCES}

Afanador, G. A., Matthews, K. A., Bartee, D., Gisselberg, J. E., Walters, M. S., Freel Meyers, C. L., et al. (2014). Redox-Dependent Lipoylation of Mitochondrial Proteins inPlasmodium Falciparum. Mol. Microbiol. 94, 156-171. doi:10.1111/ mmi. 12753

Allary, M., Lu, J. Z., Zhu, L., and Prigge, S. T. (2007). Scavenging of the Cofactor Lipoate Is Essential for the Survival of the Malaria Parasite Plasmodium Falciparum. Mol. Microbiol. 63, 1331-1344. doi:10.1111/j.13652958.2007.05592.x

Amom, Z., Zakaria, Z., Mohamed, J., Azlan, A., Bahari, H., Taufik Hidayat Baharuldin, M., et al. (2008). Lipid Lowering Effect of Antioxidant AlphaLipoic Acid in Experimental Atherosclerosis. J. Clin. Biochem. Nutr. 43, 88-94. doi:10.3164/jcbn.2008051

Biddau, M., Santha Kumar, T. R., Henrich, P., Laine, L. M., Blackburn, G. J., Chokkathukalam, A., et al. (2021). Plasmodium Falciparum LipB Mutants Display Altered Redox and Carbon Metabolism in Asexual Stages and Cannot Complete Sporogony in Anopheles Mosquitoes. Int. J. Parasitol. 51, 441-453. doi:10.1016/j.ijpara.2020.10.011

Cao, X., Hong, Y., Zhu, L., Hu, Y., and Cronan, J. E. (2018). Development and Retention of a Primordial Moonlighting Pathway of Protein Modification in the proteins are located in the mitochondria. In humans, the insertion of two sulfur atoms to generate the lipoyl moiety is catalyzed by the mitochondrial protein lipoyl synthase (LIAS) and utilizing [4Fe$4 \mathrm{~S}]$ as a co-factor.

Lipoylation events in humans remains an open research topic, since the enzymes involved in the pathway may be potential drug targets against cancer, as recently demonstrated for CPI-613 ${ }^{\circledR}$ (devimistat) (Zachar et al., 2011; Gao et al., 2020), which is currently designated as an orphan drug by FDA for the treatment of metastatic pancreatic cancer. Lipoylation event is also present in other protozoans, for example Trypanosoma cruzi (Vacchina et al., 2018), where growth of parasite in medium liver infusion tryptose (LIT) with 10 fold lower glucose concentration $(0.4 \mathrm{~g} / \mathrm{L})$ increased the lipoylated state of $\mathrm{PDH}$ E2 subunits. The LA analogue 8-bromooctanoic acid showed inhibition of parasite growth in different protozoans, including T. cruzi, P. falciparum, and Toxoplasma gondii (Crawford et al., 2006; Allary et al., 2007).

\section{AUTHOR CONTRIBUTIONS}

SY and FW wrote the manuscript, gathered literature data and provided critical suggestions. XD gathered literature data. MG provided insightful and critical revision and discussion. CW supervised the work and provided the initial suggestions.

\section{ACKNOWLEDGMENTS}

The authors would like to thank the São Paulo Research Foundation-FAPESP for providing financial support (processes 2015/26722-8, 2017/03966-4, and 2017/26358-0) and funding from the Brazilian National Council for Scientific and Technological Development-CNPq (134005/2018-0).

Absence of Selection Presents a Puzzle. Proc. Natl. Acad. Sci. USA 115, 647-655. doi:10.1073/pnas.1718653115

Cao, X., Zhu, L., Song, X., Hu, Z., and Cronan, J. E. (2018). Protein Moonlighting Elucidates the Essential Human Pathway Catalyzing Lipoic Acid Assembly on its Cognate Enzymes. Proc. Natl. Acad. Sci. USA 115, E7063-E7072. doi:10.1073/pnas.1805862115

Charan, M., Singh, N., Kumar, B., Srivastava, K., Siddiqi, M. I., and Habib, S. (2014). Sulfur Mobilization for Fe-S Cluster Assembly by the Essential SUF Pathway in the Plasmodium Falciparum Apicoplast and its Inhibition. Antimicrob. Agents Chemother. 58, 3389-3398. doi:10.1128/ AAC.02711-13

Christensen, Q. H., and Cronan, J. E. (2010). Lipoic Acid Synthesis: A New Family of Octanoyltransferases Generally Annotated as Lipoate Protein Ligases. Biochemistry 49, 10024-10036. doi:10.1021/bi101215f

Crawford, M. J., Thomsen-Zieger, N., Ray, M., Schachtner, J., Roos, D. S., and Seeber, F. (2006). Toxoplasma Gondii Scavenges Host-Derived Lipoic Acid Despite its De Novo Synthesis in the Apicoplast. EMBO J. 25, 3214-3222. doi:10.1038/sj.emboj.7601189

Deschermeier, C., Hecht, L.-S., Bach, F., Rützel, K., Stanway, R. R., Nagel, A., et al. (2012). Mitochondrial Lipoic Acid Scavenging Is Essential for Plasmodium Berghei Liver Stage Development. Cell Microbiol 14, 416-430. doi:10.1111/j.1462-5822.2011.01729.x 
Douglas, P., Kriek, M., Bryant, P., and Roach, P. L. (2006). Lipoyl Synthase Inserts Sulfur Atoms into an Octanoyl Substrate in a Stepwise Manner. Angew. Chem. Int. Ed. 45, 5197-5199. doi:10.1002/anie.200601910

Fujiwara, K., Toma, S., Okamura-Ikeda, K., Motokawa, Y., Nakagawa, A., and Taniguchi, H. (2005). Crystal Structure of Lipoate-Protein Ligase A from Escherichia C. J. Biol. Chem. 280, 33645-33651. doi:10.1074/jbc.M505010200

Gao, L., Xu, Z., Huang, Z., Tang, Y., Yang, D., Huang, J., et al. (2020). CPI-613 Rewires Lipid Metabolism to Enhance Pancreatic Cancer Apoptosis via the AMPK-ACC Signaling. J. Exp. Clin. Cancer Res. 39, 73. doi:10.1186/ s13046-020-01579-x

Grayczyk, J. P., and Alonzo, F. (2019). Staphylococcus A Lipoic Acid Synthesis Limits Macrophage Reactive Oxygen and Nitrogen Species Production to Promote Survival during Infection. Infect. Immun. 87, e00344-19. doi:10.1128/IAI.00344-19

Grayczyk, J. P., Harvey, C. J., Laczkovich, I., and Alonzo, F. (2017). A Lipoylated Metabolic Protein Released by Staphylococcus A Suppresses Macrophage Activation. Cell Host \& Microbe 22, 678-687. e9. doi:10.1016/ j.chom.2017.09.004

Günther, S., Matuschewski, K., and Müller, S. (2009). Knockout Studies Reveal an Important Role of Plasmodium Lipoic Acid Protein Ligase A1 for Asexual Blood Stage Parasite Survival. PLoS One 4, e5510. doi:10.1371/ journal.pone.0005510

Günther, S., Storm, J., and Müller, S. (2009). Plasmodium Falciparum: Organelle-Specific Acquisition of Lipoic Acid. Int. J. Biochem. Cel Biol. 41, 748-752. doi:10.1016/j.biocel.2008.10.028

Hane, F., and Leonenko, Z. (2014). Effect of Metals on Kinetic Pathways of Amyloid- $\beta$ Aggregation. Biomolecules 4, 101-116. doi:10.3390/ biom 4010101

Jumper, J., Evans, R., Pritzel, A., Green, T., Figurnov, M., Ronneberger, O., et al. (2021). Highly Accurate Protein Structure Prediction with AlphaFold. Nature 596, 583-589. doi:10.1038/s41586-021-03819-2

Koes, D. R., Baumgartner, M. P., and Camacho, C. J. (2013). Lessons Learned in Empirical Scoring with Smina from the CSAR 2011 Benchmarking Exercise. J. Chem. Inf. Model. 53, 1893-1904. doi:10.1021/ci300604z

Kozakov, D., Grove, L. E., Hall, D. R., Bohnuud, T., Mottarella, S. E., Luo, L., et al. (2015). The FTMap Family of Web Servers for Determining and Characterizing Ligand-Binding Hot Spots of Proteins. Nat. Protoc. 10, 733-755. doi:10.1038/nprot.2015.043

Laczkovich, I., Teoh, W. P., Flury, S., Grayczyk, J. P., Zorzoli, A., and Alonzo, F. (2018). Increased Flexibility in the Use of Exogenous Lipoic Acid byStaphylococcus Aureus. Mol. Microbiol. 109, 150-168. doi:10.1111/ mmi.13970

Leung, K.-Y., De Castro, S. C. P., Galea, G. L., Copp, A. J., and Greene, N. D. E. (2021). Glycine Cleavage System H Protein Is Essential for Embryonic Viability, Implying Additional Function beyond the Glycine Cleavage System. Front. Genet. 12, 625120. doi:10.3389/fgene.2021.625120

Li, W., Yin, L., Sun, X., Wu, J., Dong, Z., Hu, K., et al. (2020). Alpha-Lipoic Acid Protects against Pressure Overload-Induced Heart Failure via ALDH2-dependent Nrf1-FUNDC1 Signaling. Cell Death Dis 11, 599. doi:10.1038/s41419-020-02805-2

Marangon, K., Devaraj, S., Tirosh, O., Packer, L., and Jialal, I. (1999). Comparison of the Effect of a-lipoic Acid and $\alpha$-tocopherol Supplementation on Measures of Oxidative Stress. Free Radic. Biol. Med. 27, 1114-1121. doi:10.1016/S0891-5849(99)00155-0

McFadden, G. I., and Yeh, E. (2017). The Apicoplast: Now You See it, Now You Don't. Int. J. Parasitol. 47, 137-144. doi:10.1016/j.ijpara.2016.08.005

McNeilly, A. M., Davison, G. W., Murphy, M. H., Nadeem, N., Trinick, T., Duly, E., et al. (2011). Effect of a-lipoic Acid and Exercise Training on Cardiovascular Disease Risk in Obesity with Impaired Glucose Tolerance. Lipids Health Dis. 10, 217. doi:10.1186/1476-511X-10-217

Molz, P., de Freitas, B. S., Uberti, V. H., da Costa, K. M., Kist, L. W., Bogo, M. R., et al. (2021). Effects of Lipoic Acid Supplementation on Age- and IronInduced Memory Impairment, Mitochondrial DNA Damage and Antioxidant Responses. Eur. J. Nutr. 60, 3679-3690. doi:10.1007/s00394021-02541-z

Oppenheim, R. D., Creek, D. J., Macrae, J. I., Modrzynska, K. K., Pino, P., Limenitakis, J., et al. (2014). BCKDH: The Missing Link in Apicomplexan Mitochondrial Metabolism Is Required for Full Virulence of Toxoplasma
Gondii and Plasmodium Berghei. Plos Pathog. 10, e1004263. doi:10.1371/ journal.ppat. 1004263

Pala, Z. R., Saxena, V., Saggu, G. S., Mani, S. K., Pareek, R. P., Kochar, S. K., et al. (2019). Functional Analysis of Iron-Sulfur Cluster Biogenesis (SUF Pathway) from Plasmodium Vivax Clinical Isolates. Exp. Parasitol. 198, 53-62. doi:10.1016/j.exppara.2019.01.015

Pietikäinen, L. P., Rahman, M. T., Hiltunen, J. K., Dieckmann, C. L., and Kastaniotis, A. J. (2021). Genetic Dissection of the Mitochondrial Lipoylation Pathway in Yeast. BMC Biol. 19, 14. doi:10.1186/s12915-02100951-3

Rack, J. G. M., Morra, R., Barkauskaite, E., Kraehenbuehl, R., Ariza, A., Qu, Y., et al. (2015). Identification of a Class of Protein ADP-Ribosylating Sirtuins in Microbial Pathogens. Mol. Cel 59, 309-320. doi:10.1016/ j.molcel.2015.06.013

Shears, M. J., Botté, C. Y., and McFadden, G. I. (2015). Fatty Acid Metabolism in the Plasmodium Apicoplast: Drugs, Doubts and Knockouts. Mol. Biochem. Parasitol. 199, 34-50. doi:10.1016/j.molbiopara.2015.03.004

Smirnova, J., Kabin, E., Järving, I., Bragina, O., Tõugu, V., Plitz, T., et al. (2018). Copper(I)-Binding Properties of De-Coppering Drugs for the Treatment of Wilson Disease. $\alpha$-Lipoic Acid as a Potential Anti-Copper Agent. Sci. Rep. 8, 1463. doi:10.1038/s41598-018-19873-2

Spalding, M. D., and Prigge, S. T. (2010). Lipoic Acid Metabolism in Microbial Pathogens. Microbiol. Mol. Biol. Rev. 74, 200-228. doi:10.1128/ MMBR.00008-10

Storm, J., and Müller, S. (2012). Lipoic Acid Metabolism of Plasmodium - A Suitable Drug Target. Curr. Pharm. Des. 18, 3480-3489. doi:10.2174/ 138161212801327266

Tang, Q., Guo, Y., Meng, L., and Chen, X. (2021). Chemical Tagging of Protein Lipoylation. Angew. Chem. Int. Ed. 60, 4028-4033. doi:10.1002/anie.202010981

Teoh, W. P., Chen, X., Laczkovich, I., and Alonzo, F. (2021). Staphylococcus A Adapts to the Host Nutritional Landscape to Overcome Tissue-Specific Branched-Chain Fatty Acid Requirement. Proc. Natl. Acad. Sci. USA 118, e2022720118. doi:10.1073/pnas.2022720118

Teoh, W. P., Resko, Z. J., Flury, S., and Alonzo, F. (2019). Dynamic Relay of Protein-Bound Lipoic Acid in Staphylococcus A. J. Bacteriol. 201, e00446-19. doi:10.1128/JB.00446-19

Thomsen-Zieger, N., Schachtner, J., and Seeber, F. (2003). Apicomplexan Parasites Contain a Single Lipoic Acid Synthase Located in the Plastid. FEBS Lett. 547, 80-86. doi:10.1016/S0014-5793(03)00673-2

Tromba, L., Perla, F., Carbotta, G., Chiesa, C., and Pacifico, L. (2019). Effect of Alpha-Lipoic Acid Supplementation on Endothelial Function and Cardiovascular Risk Factors in Overweight/Obese Youths: A Double-Blind, Placebo-Controlled Randomized Trial. Nutrients 11, 375. doi:10.3390/ nu11020375

Turner, N. A., Sharma-Kuinkel, B. K., Maskarinec, S. A., Eichenberger, E. M., Shah, P. P., Carugati, M., et al. (2019). Methicillin-Resistant Staphylococcus A: An Overview of Basic and Clinical Research. Nat. Rev. Microbiol. 17, 203-218. doi:10.1038/s41579-018-0147-4

Vacchina, P., Lambruschi, D. A., and Uttaro, A. D. (2018). Lipoic Acid Metabolism in Trypanosoma Cruzi as Putative Target for Chemotherapy. Exp. Parasitol. 186, 17-23. doi:10.1016/j.exppara.2018.01.017

van Hal, S. J., Jensen, S. O., Vaska, V. L., Espedido, B. A., Paterson, D. L., and Gosbell, I. B. (2012). Predictors of Mortality in Staphylococcus A Bacteremia. Clin. Microbiol. Rev. 25, 362-386. doi:10.1128/CMR.05022-11

van Schaijk, B. C. L., Kumar, T. R. S., Vos, M. W., Richman, A., van Gemert, G.-J., Li, T., et al. (2014). Type II Fatty Acid Biosynthesis Is Essential for Plasmodium Falciparum Sporozoite Development in the Midgut of Anopheles Mosquitoes. Eukaryot. Cel 13, 550-559. doi:10.1128/EC.00264-13

Vaughan, A. M., O’Neill, M. T., Tarun, A. S., Camargo, N., Phuong, T. M., Aly, A. S. I., et al. (2009). Type II Fatty Acid Synthesis Is Essential Only for Malaria Parasite Late Liver Stage Development. Cel Microbiol 11, 506-520. doi:10.1111/ j.1462-5822.2008.01270.x

Wang, M., Wang, Q., Gao, X., and Su, Z. (2017). Conditional Knock-Out of Lipoic Acid Protein Ligase 1 Reveals Redundancy Pathway for Lipoic Acid Metabolism in Plasmodium Berghei Malaria Parasite. Parasites Vectors 10, 315. doi:10.1186/ s13071-017-2253-y

WHO (2020). Report on Antimalarial Drug Efficacy, Resistance and Response: 10 Years of Surveillance (2010-2019). Geneva, Switzerland: WHO. 
Wiley, J. D., Merino, E. F., Krai, P. M., McLean, K. J., Tripathi, A. K., VegaRodríguez, J., et al. (2015). Isoprenoid Precursor Biosynthesis Is the Essential Metabolic Role of the Apicoplast during Gametocytogenesis in Plasmodium Falciparum. Eukaryot. Cel 14, 128-139. doi:10.1128/EC.00198-14

World Health Organization (2021). Malaria - Key Facts. World Heal Organ. Available at: https://www.who.int/news-room/fact-sheets/detail/malaria (accessed June 16, 2021).

World Health Organization (2020). World Malaria Report 2020: 20 Years of Global Progress and Challenges. Geneva, Switzerland: World Health Organization, Licence: CC BY-NC-SA 3.0 IGO.

Wrenger, C., and Müller, S. (2004). The Human Malaria Parasite Plasmodium Falciparum Has Distinct Organelle-specific Lipoylation Pathways. Mol. Microbiol. 53, 103-113. doi:10.1111/j.13652958.2004.04112.x

Xiao, Y., Cui, J., Shi, Y., and Le, G. (2012). Alpha-Lipoic Acid Protects against Hydrogen Peroxide-Induced Oxidative Stress in MC3T3-E1 OsteoblastLike Cells. J. Funct. Foods 4, 642-649. doi:10.1016/j.jff.2012.04.004

Zachar, Z., Marecek, J., Maturo, C., Gupta, S., Stuart, S. D., Howell, K., et al. (2011). Non-Redox-Active Lipoate Derivates Disrupt Cancer Cell Mitochondrial Metabolism and Are Potent Anticancer Agents In Vivo. J. Mol. Med. 89, 1137-1148. doi:10.1007/s00109-011-0785-8

Zhang, X., Nie, J., Zheng, Y., Ren, J., and Zeng, A. P. (2020). Activation and Competition of Lipoylation of $\mathrm{H}$ Protein and its Hydrolysis in a Reaction cascade Catalyzed by the Multifunctional Enzyme Lipoate-Protein Ligase A. Biotechnol. Bioeng. 117, 3677-3687. doi:10.1002/bit.27526

Zorzoli, A., Grayczyk, J. P., and Alonzo, F. (2016). Staphylococcus A Tissue Infection during Sepsis Is Supported by Differential Use of Bacterial or Host-Derived Lipoic Acid. Plos Pathog. 12, e1005933. doi:10.1371/journal.ppat.1005933

Conflict of Interest: The authors declare that the research was conducted in the absence of any commercial or financial relationships that could be construed as a potential conflict of interest.

Publisher's Note: All claims expressed in this article are solely those of the authors and do not necessarily represent those of their affiliated organizations, or those of the publisher, the editors and the reviewers. Any product that may be evaluated in this article, or claim that may be made by its manufacturer, is not guaranteed or endorsed by the publisher.

Copyright (ङ 2021 Rei Yan, Wakasuqui, Du, Groves and Wrenger. This is an openaccess article distributed under the terms of the Creative Commons Attribution License (CC BY). The use, distribution or reproduction in other forums is permitted, provided the original author(s) and the copyright owner(s) are credited and that the original publication in this journal is cited, in accordance with accepted academic practice. No use, distribution or reproduction is permitted which does not comply with these terms. 